\title{
Silicone Gel for Scar Prevention
}

Thomas A. Mustoe

\section{Contents}

\subsection{History - 204}

23.2 The Role of the Epithelium in Scar Formation - 204

23.2.1 Delay in Epithelization - 204

23.2.2 Clinical Studies - 206

23.2.3 Sheets Versus Creams - 207

Bibliography - 208 


\subsection{History}

Silicone gel was first discovered to be useful in the treatment of scars by Perkins in Australia and New Zealand when observations were made empirically that scars improved when silicone was used as part of a dressing. The observations although anecdotal were impressive enough that use spread to the UK, and at the University of Strathclyde in Glasgow, Scotland, a biomedical engineering student, Karen Quinn, undertook a series of studies for her $\mathrm{PhD}$ thesis to determine the mechanism of action and published a paper in burns [1]. She determined that neither heat nor pressure nor silicone absorption or chemical effects were responsible for its effects in studies done together with surgeon from the Plastic Surgery Unit at Canniesburn (Professor Reid). By chance, I was visiting the Canniesburn Hospital for 2 weeks and had the opportunity to observe the use of silicone gel, which was made by Dow Corning. They were already sponsoring some of my studies at Washington University School of Medicine, and I suggested a controlled clinical study which had not yet been done. The effects on immature hypertrophic scars were in some cases dramatic with each patient serving as their own control. We subsequently did a follow-up study on using silicone on very early scars to prevent hypertrophy with equally encouraging results. Although there was some skepticism, in part due to a lack of a clear mechanism of action, subsequent studies by several other investigators confirmed the efficacy. I will detail some of these studies later in this chapter.

\subsection{The Role of the Epithelium in Scar Formation}

Hypertrophic scars are due to an excess of collagen deposition which is almost entirely from fibroblasts. The role of excess inflammation, myofibroblasts, and other fibroblasts phenotypically that produce excess collagen, tension, and genetic factors all play a role in hypertrophic scar formation. Given that silicone is not absorbed into the skin and covers intact epithelium, the beneficial effects must be indirect through effects on the epithelium.

The epithelium serves an important barrier function. Water loss through the epithelium is limited by a functional stratum corneum, and disturbances in barrier function are associated in many dermatologic conditions with increased inflammation instigated by inflammatory growth factors and other inflammatory mediators produced by epidermal cells. Epithelial mesenchymal cell cross talk is well known.

\subsubsection{Delay in Epithelization}

When barrier function is disrupted by tissue injury, restoration of the stratum corneum lags behind epithelization which is initially only one cell layer thick as epithelium rapidly migrates over an open wound, followed by stratification, followed eventually by a multilayered stratum corneum. However, there is evidence that the immature stratum corneum does not become fully functional as a water barrier for weeks to months. During that time, there is a stimulus to restore homeostatic barrier function by increased epithelial proliferation, as manifested by increases in thickness, alterations in differentiation markers in the immature differentiating epithelium, and increased inflammatory mediator synthesis [2].

In cell culture, prevention of water loss by a liquid or high-humidity environment results in a reduction in soluble inflammatory mediators and consequently a reduction in collagen synthesis in a coculture setup with fibroblasts $[3,4]$. In vivo, in models in rabbits, rats, and mice, prevention of water loss from an epithelium with an immature, deficient stratum corneum results in increase in inflammatory mediators similar to those utilizing human cells in an in vitro system [5]. There are multiple highly upregulated factors including IL-1, IL-8, and Cox-2. In total, over 1000 genes are either upregulated or downregulated in an environment where transepidermal water loss (TEWL) is limited by a semiocclusive dressing versus no covering [6].

Humans are almost unique in their tendency to heal with excessive or hypertrophic scarring. In part this is due to the "tight-skinned" characteristic of the human skin which is tightly adherent to the underlying muscle layer due to the lack of a panniculus carnosus. Thus, human wounds heal only part by wound contracture, with both a delay in epithelization and also tension forces due to the constraints on skin contraction by adherence to the underlying deeper tissues. The rabbit ear [7] mimics human skin in being firmly adherent to the underlying cartilage which splints the wound and minimizes contracture. Healing is almost entirely due to epithelization, and with wounds larger than $6 \mathrm{~mm}$ in diameter, healing is delayed beyond 2 weeks which in humans is the critical delay in healing sufficient to increase the risk of hypertrophic scars. In addition, during the healing process, the fibroblasts in the granulation tissue are under tension because the contractile forces generated by the myofibroblasts are counteracted by the resistance to contraction by the stiff underlying cartilage. Wounds on the rabbit ear heal with scar elevation (• Fig. 23.1) which both from gross visual appearance, and also histologically closely mimic human hypertrophic scars. Larger wounds with epithelization delayed beyond 2 weeks have 


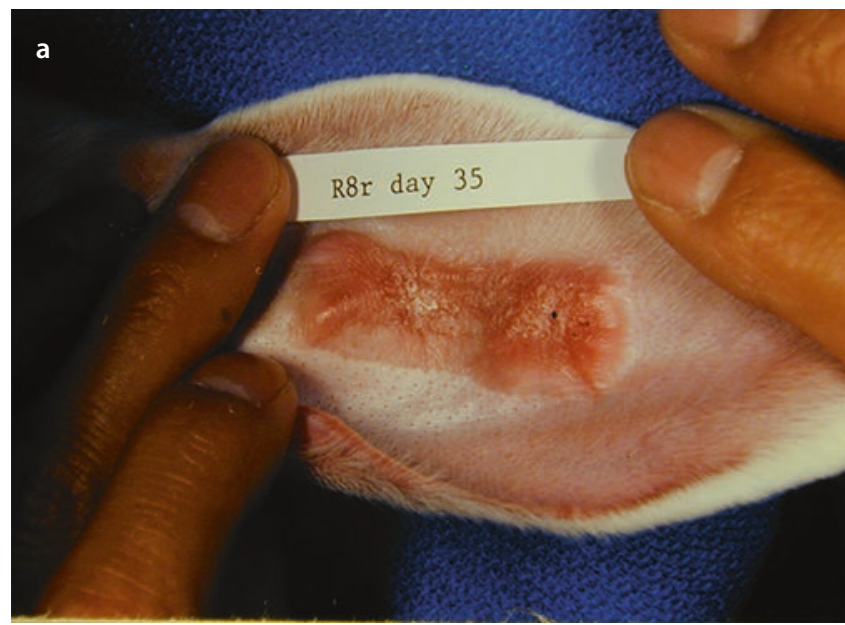

- Fig. 23.1 a A large scar in the rabbit ear created by full-thickness removal of the skin followed by healing without dressings. b By creating small 6-8 $\mathrm{mm}$ in diameter full-thickness wounds with a circular

greater scar elevation, and like humans, older rabbits heal with flatter scars. The scars respond to interventions like local steroid and injections and blockers of fibrosis including TGFB antibodies and antisense to CTGF, in which both growth factors induce collagen synthesis and are upregulated in fibrotic processes. Silicone gel sheets and silicone cream both reduce scarring, and other semiocclusive agents such as paper tape, polyurethane films, cyanoacrylates, or stoma adhesive are also effective which implicates occlusion as the working mechanism of silicone gel, when combined with negative data ruling out absorption (chemical process) or heat or pressure.

We have used this model to investigate the working mechanism of silicone gel or other occlusive dressings in a series of experiments. In our model, silicone gel resulted in decreased inflammatory cytokines including IL-1, TNF, IL-8, and Cox-2 among others. The net effect of increased hydration is a decrease in osmolarity and several extracellular fluid ions including calcium, sodium, and chloride. By controlling otherwise for potential ions and osmolarity, we found that $\mathrm{Na}$ concentration was the critical factor controlling signaling for the inflammatory cytokines affected by hydration. We also found that changes in extracellular $\mathrm{Na}$ concentration resulted in changes in transmembrane $\mathrm{Na}$ flux which is mediated chiefly by the $\mathrm{Na}$ channel ENaC. Briefly, an increase in $\mathrm{Na}$ concentration due to increased TEWL caused by a deficient epithelial barrier results in activation of $\mathrm{ENaC}$ which controls levels of COX-2 resulting in increased prostaglandins [3].

The question remained of how increases in $\mathrm{Na}$ concentration are sensed resulting in activation of $\mathrm{ENaC}$. We looked for changes in all of the candidate $\mathrm{Na}$ channels in epitheilia cells that could conceivably respond to changes in $\mathrm{Na}$ concentration and found only one channel, Nax, had a significant response. Nax is a

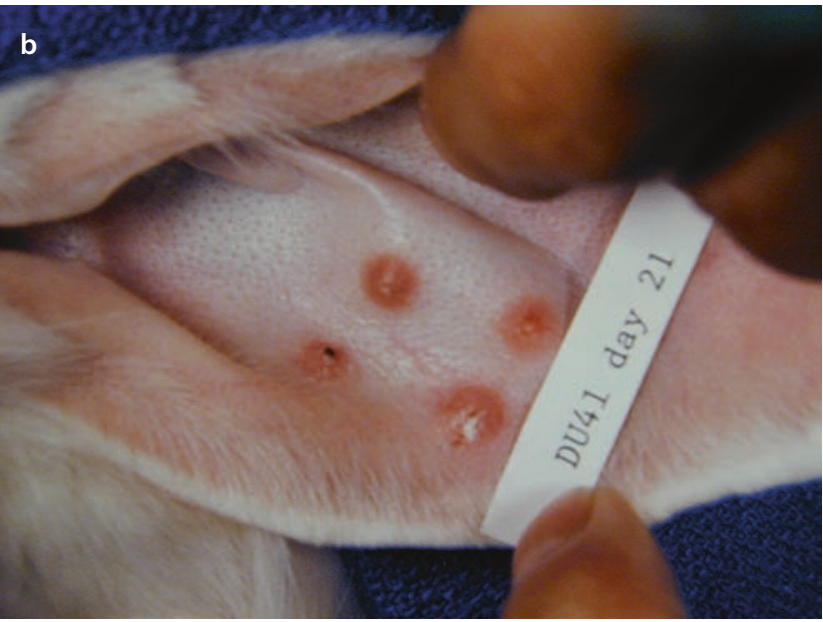

punch, healing is complete in about 2 weeks and the scar is elevated allowing quantification. This rabbit ear model has been used for all of our experimental studies

member of the voltage-gated $\mathrm{Na}$ channels but lacks the extracellular domain responsive for voltage gating. It's function previously has been unclear. $\mathrm{NaX}$ has been found to be Na concentration sensor in the central nervous system, but its function and presence in other organs and cell types had not been extensively studied. With a series of knockdown experiments in vitro, we established that $\mathrm{NaX}$ controls the entire signal transduction signaling seen with changes in $\mathrm{Na}$ concentration and hydration in vitro in epithelial cells and also skin explants. Utilizing our model of hypertrophic scarring in the rabbit ear, we found treatment with antisense to $\mathrm{NaX}$ results in a very significant reduction in scarring, similar or greater to the effects of silicone gel. Furthermore blocking downstream signals including Cox-2, IL-B, and the S-100 proteins also reduced scarring. Blocking $\mathrm{ENaC}$ with a clinically used pharmacologic agent, amiloride, also resulted in decreased scarring. Experiments to elucidate the signal transduction pathways further have established that $\mathrm{NaX}$ responds or senses high salt with activation of a serine protease, prostasin, which activates $\mathrm{ENaC}$. The importance of $\mathrm{NaX}$ in controlling changes in the epithelium includes profound changes in epithelial differentiation, cell migration, and cell proliferation in knockdown experiments in vitro [4].

In summary the application of silicone gel to an acute scar with a deficient epithelial water barrier due to an immature stratum corneum results in decreased water loss (TEWL). This prevents the increase in Na concentration which otherwise results in an $\mathrm{NaX}$-mediated complex signal transduction pathway which includes downstream activation of $\mathrm{ENaC}$ as well as hundreds of other genes, mirroring the kind of broad changes in gene expression found to be controlled by many cyto- 
kines such as TGFB. Other occlusive treatments are also effective, but the degree of occlusion is important (complete occlusion results in excessive hydration with impacts on surface bacterial levels as well as undesirable physical changes in the epithelium).

\subsubsection{Clinical Studies}

The first controlled clinical study to demonstrate efficacy of silicone gel utilized silicone gel sheets applied to portions of immature hypertrophic scars due to burns, trauma, or surgery, in an effort to treat immature hypertrophic scars (- Fig. 23.2). Treatment went on for 3 months with application of the gel for 12 hours. We found that 24-hour treatment led to maceration and was poorly tolerated. One of the challenges was to quantify the improvement in scarring. Although photographic evidence is essential, rating scar severity remains challenging and is ultimately nonquantitative. In an effort to have a completely objective and quantitative method of measuring scar severity, we turned to measuring the scar stiffness. Scars are stiffer (less elastic) than normal skin, and as scars improve they become more pliable or more elastic. Utilizing an extensometer which measures the amount of stretch when the skin is subjected to a given force, we were able to calculate Young's modulus and get an objective measure of scar stiffness. We first used an extensometer to measure the stiffness of burn scars and found that older scars were more elastic and there was a very good correlation with age of burn scar to stiffness of the scar over several years [8]. In our scar study, we found a highly statistically significant reduction in stiffness that reached closed to its maximum after 2 months of treatment, with only minor changes by extending treatment for 3 months [9]. Furthermore, the improve- ment was sustained after treatment was terminated. At the same time, there were a substantial reduction in scar erythema and flattening of the scar clinically which was obvious on photographs. We saw no response in a keloid and much more impressive results in immature scars, with minor effects or no effect on mature scars (scars older than 1 year without erythema).

In a follow-up study (• Fig. 23.3), we wanted to see if we could prevent the development of hypertrophic scars by beginning treatment early on surgical incisional scars. Again, a portion of the incision was treated with an adjacent area untreated [10]. We again utilized photography but wanted to quantify the scar volumes for an objective quantitative measure. Dental alginate was used to make a negative impression which was then converted to a positive impression using dental plaster in similar fashion to the way dentists take dental impressions for models used in a variety of dental and oral surgical procedures. The positive molds there then burred down to a totally flat surface and volumes calculated by measuring the difference in weights. Treated areas of the scars were found to have significantly less volumes as long as the untreated scar developed some hypertrophy. Not surprisingly, in scars that healed favorably, minimal or no differences were seen.

Initially, although these studies received considerable attention, even in the popular press, there was some skepticism by many clinicians, due in part to the lack of mechanism. Over the subsequent years, many studies have confirmed the observations we made relying on subjective evaluation of scar severity using a variety of scar severity scales such as the Vancouver Scar Scale and Patient and Observer Scar Assessment Scale (POSAS). In 2002, an international group developed an evidencebased analysis to develop guidelines for treatment [11] and found that there was strong evidence for the use of
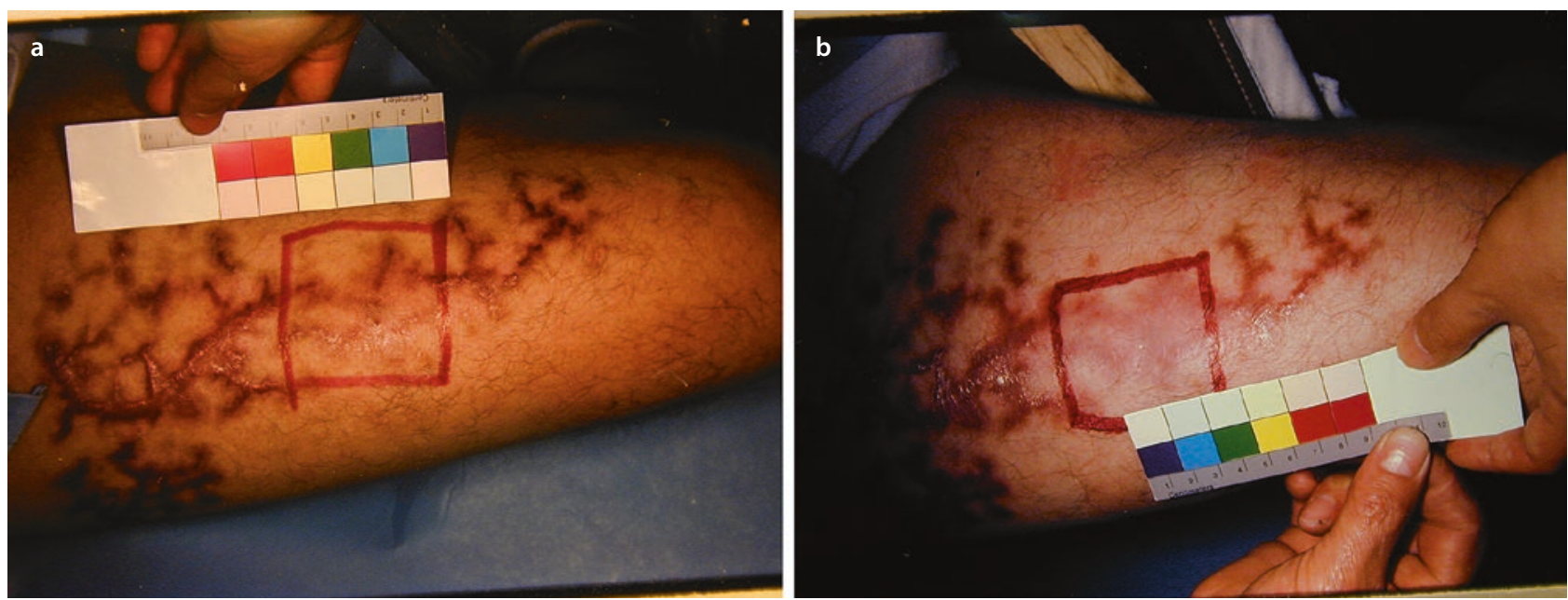

- Fig. 23.2 a Partial-thickness immature hypertrophic burn scar. The square marks the planned treatment area with silicone gel. b After 2 months of treatment, the treated area is flatter, more pliable, and less pink 


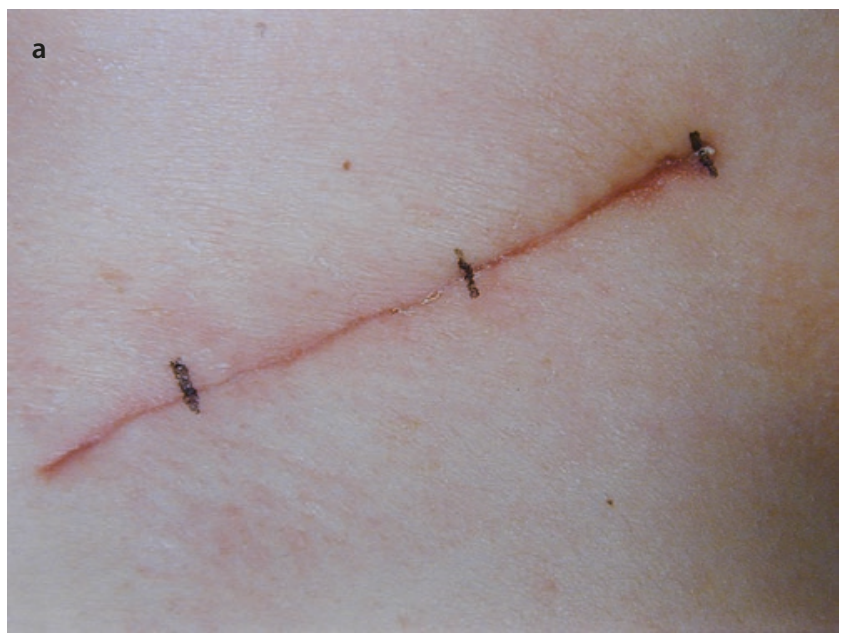

- Fig. 23.3 a A fresh surgical incision prior to treatment. One area of the scar is going to be treated preventively with the other portion of the scar serving as comparison. b After 2 months, the

silicone gel both for the treatment and prevention of hypertrophic scars.

Since 2002, many more studies have been published on silicone gel with supportive data. Most of these studies were not prospective randomized trials and by the nature of the treatment virtually impossible to blind. The Cochrane group did an analysis and noted the deficiencies of the studies and made the conclusion that solid evidence for silicone gel was lacking but failed to recognize the strengths of our early studies in which the patients served as their own control and quantitative objective data was collected.

\subsubsection{Sheets Versus Creams}

There are some limitations to silicone gel sheets. They are not completely self-adhering with some attachment method such as a covering bandage, although clothing can be used in some situations (such as a bra) to aid in fixation. In tropical climates with high humidity, the scar can develop a "heat" rash due to excessive moisture underneath the gel. The gel sheeting can pick up dirt and perspiration which makes cleaning or using new sheets essential. As a practical matter, patients have difficulty wearing the sheets 24 hours/day. Our original studies suggested that using the sheets to cover the scars needed to be utilized at least 12 hours/day to be effective.

Silicone gel creams have been alternative products that address many of the limitations of silicone gel sheeting. They can be used in tropical climates more easily, do not require any additional adherence methodology, and are easier to apply 24 hours/day with twice-daily applications. The question arises whether they are as effective. One potential limitation is the chance that the

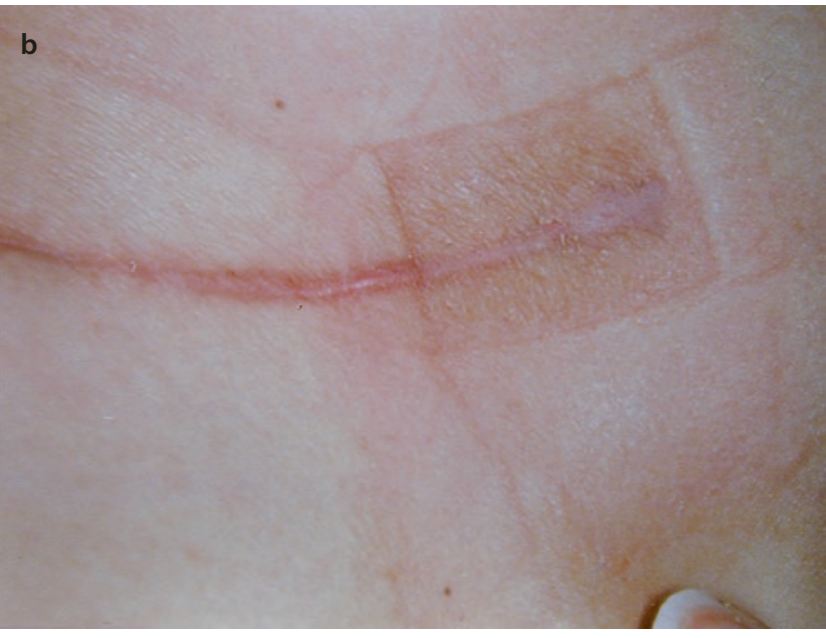

untreated scar is slightly elevated and pink in color. The area of the scar treated with silicone gel sheeting is flatter and less pink in color

cream will be rubbed off. Some silicone creams dry in place and are less likely to rub off.

We have addressed the question of efficacy of silicone gel creams in our rabbit ear animal model of hypertrophic scarring and have found the cream to be as effective as sheeting in multiple studies. All of our studies investigating the mechanism of silicone gel that we referred to earlier utilized silicone gel cream because of its ease of use.

Multiple well-done clinical studies have been done over the last 15 years that have confirmed efficacy of silicone gel. In Asian patients who are particularly high risk for hypertrophic scars, Chan [12] performed a randomized, double-blind controlled study in 50 Asian patients with a sternotomy wound following coronary bypass surgery or cardiac valvular surgery. They found that silicone gel significantly reduced multiple scar attributes including visual parameter pigmentation, vascularity, pliability, height, as well as symptoms of pain and itchiness compared with control wounds ( $p \leq 0.02$ for all). No treatment-related adverse effects were reported. To date, no comparison studies have directly compared the efficacy of sheeting to silicone gel cream. Due to the very thin film formed by silicone gel cream and the uncertainty of how effectively it remains in place when subjected to rubbing by clothing or movement, it is quite possible or even likely that sheeting might be more effective, but in practice this may be offset by the ease in 24 hours/day use.

Recently a meta-analysis of all prospective randomized controlled trials as well as additional controlled studies between 1990 and 2014 found a total of 11 studies that qualified involving 864 patient scars in which the silicone gel was used to prevent scars. They found a highly positive result from silicone gel or silicone gel sheeting particularly in patients at high risk, but even 
when all patient were included, the results were statistically significant $(p<0.02)[13]$.

In summary, silicone gel sheeting has an almost 40-year history, with widespread clinical use for 20 years. Our laboratory and others have found a compelling mechanism based on the semiocclusive properties of silicone gel, which normalize transepidermal water loss in scars with a deficient stratum corneum barrier function, and via the $\mathrm{Na}$ channels $\mathrm{ENaC}$ and $\mathrm{Nax}$ regulated.

\section{Bibliography}

1. Quinn KJ, Evans JH, Courtney JM, Gaylor JD, Reid WH. Nonpressure treatment of hypertrophic scars. Burns Incl Therm Inj. 1985;12:102-8.

2. O' Shaughnessey K, Roy N, Mustoe TA. Homeostasis of the epidermal barrier layer: a theory of how occlusion decreases hypertrophic scarring. Wound Repair Regen. 2009;17:700-8.

3. Xu W, Hong SJ, Zeitchek M, Cooper G, Jia S, Ping X, Quresh HA, Zhong A, Porterfield MD, Galiano RD, Surmeier DJ, Mustoe TA. Hydration status regulates sodium flux and inflammatory pathways through epithelial sodium channel $(\mathrm{ENaC})$ in skin. J Invest Dermatol. 2015;135(3):796-806.

4. Xu W, Hong SJ, Zhong A, Xie P, Jia S, Xie Z, Zeitchek M, Niknam-Bienia S, Zhao J, Porterfield DM, Surmeier DJ, Leung $\mathrm{KP}$, Galiano RD, Mustoe TA. Sodium channel $\mathrm{Na}_{\mathrm{x}}$ is a regulator in epithelial sodium homeostasis. Sci Transl Med. 2015;7 (312):312ra177
5. Gallant-Behm CL, Du P, Lin S, Marucha PT, DiPietro LA, Mustoe TA. Epithelial regulation of mesenchymal tissue behavior. J Investigative Dermatology. 2011;131:892-9. Pubmed ID 21228814

6. Xu W, Jia S, Xie P, Zhong A, Galiano RD, Mustoe TA, Hong SJ. The expression of Proinflammatory genes in epidermal keratinocytes is regulated by hydration status. J Invest Dermatol. 2013;134:1044-55.

7. Morris DD, Zhao LL, Bolton L, Roth SI, Ladin DA, Mustoe TA. Acute and chronic models for excessive dermal scarring: quantitative studies. Plast Reconstr Surg. 1997;100:674-81.

8. Bartell TH, Monafo WW, Mustoe TA. Noninvasive in vivo quantification of elastic properties of hypertrophic scar: hand held elastometry. J Burn Care Rehabil. 1988;9:657-60.

9. Ahn ST, Monafo W, Mustoe TA. Topical silicone gel: a new treatment for hypertrophic scars. Surgery. 1989;106:781-7.

10. Ahn ST, Monafo WW, Mustoe TA. Topical silicone gel for the prevention and treatment of hypertrophic scar. Arch Surg. 1991;126:499-504.

11. Mustoe TA, Cooter R, Gold M, Hobbs R, Ramelet AA, Shakespeare P, Stella M, Teot L, Wood F, Ziegler U. International clinical guidelines for scar management. Plastic and Reconstr Surg. 2002;110:560-72.

12. Chan KY, Lau CL, Adeeb SM, Somasundaram S, Nasir-Zahari M. A randomized, placebo-controlled, double-blind, prospective clinical trial of silicone gel in prevention of hypertrophic scar development in median sternotomy wound. Plast Reconstr Surg. 2005;116(4):1013-20.

13. Hsu K-C, Luan C-W, Tsai Y-W. Review of silicone gel sheeting and silicone gel for the prevention of hypertrophic scars and keloids wounds. 2017;29:154-8.

Open Access This chapter is licensed under the terms of the Creative Commons Attribution 4.0 International License (http://creativecommons. $\mathrm{org} /$ licenses/by/4.0/), which permits use, sharing, adaptation, distribution and reproduction in any medium or format, as long as you give appropriate credit to the original author(s) and the source, provide a link to the Creative Commons license and indicate if changes were made.

The images or other third party material in this chapter are included in the chapter's Creative Commons license, unless indicated otherwise in a credit line to the material. If material is not included in the chapter's Creative Commons license and your intended use is not permitted by statutory regulation or exceeds the permitted use, you will need to obtain permission directly from the copyright holder. 